\title{
TEM and HRTEM characterization of nanocomposites reinforced with carbon nanotubes
}

\author{
S. Simões ${ }^{*}$, F. Viana*, M. A. L. Reis ${ }^{* *}$, M. F. Vieira ${ }^{*}$ \\ * CEMUC, Department of Metallurgical and Materials Engineering, University of Porto, R. Dr. Roberto \\ Frias, 4200-465 Porto, Portugal \\ *** Faculdade de Ciências Exatas e Tecnologia, Universidade Federal do Pará, Abaetetuba, PA 68440-000 \\ Brazil
}

Email: ssimoes@fe.up.pt

The extraordinary properties of carbon nanotubes (CNT), as high stiffness (970 GPa), high strength (63 GPa) and high thermal conductivity, combined with its low weight [1], have generated a great attention from the research community. The use of CNT as reinforcement material for metal matrix composites has been explored in recent years due to the potential hardening effect of these hard and stiff nanomaterials [2]. Metal matrix nanocomposites are excellent candidates for various applications due to high strength and stiffness, desirable coefficient of thermal expansion and good damping properties.

This investigation focus on the production of aluminium matrix composites reinforced with CNT, by powder metallurgy techniques. CNT used in this work (from FIBERMAX composites) are mostly multi-walled CNT agglomerated in large clusters. The characterization of CNT was performed by scanning and transmission electron microscopy (SEM and TEM) and high resolution transmission electron microscopy (HRTEM). The dispersion of CNT was carried out using an ultrasonicator during 15 minutes in isopropanol. Figure 1 shows that this dispersion treatment was effective to untangle the CNT clusters. To produce the composites, Al powders (from Goodfellow), with a maximum particle size of $50 \mu \mathrm{m}$ and a purity of $99.5 \%$, were mixed with CNT in a Turbula during $1 \mathrm{~h}$. Mixtures of Al powders and CNT ( 0 to $2 \mathrm{wt} . \%$ ) were hot pressed and sintered at $600{ }^{\circ} \mathrm{C}$ during $90 \mathrm{~min}$ under a pressure of $50 \mathrm{MPa}$, with a vacuum of $10^{-2} \mathrm{~Pa}$. Microstructural characterization of CNT/Al composites was performed by SEM, TEM and HRTEM. SEM and TEM images of the nanocomposites revealed a uniform dispersion of carbon nanotubes throughout the aluminium matrix for $0.75 \mathrm{wt} . \%$ of CNT. The CNT are observed through the composite at grain boundary junctions, as expected, but also in the matrix. TEM images of the nanocomposites produced with 0.75 and 1.0 wt. \% of CNT are present in Figure 2. In these images it is possible to observe CNT inside the aluminium grains, HRTEM observation (Fig. 3) revealed that these CNT are well bonded to the aluminium matrix. A strong interface between CNT and matrix is essential to an effective load transfer. The formation of $\mathrm{Al}_{4} \mathrm{C}_{3}$ phase, due to the reaction between the $\mathrm{CNT}$ and the $\mathrm{Al}$, was also identified by HRTEM observations.

This research is sponsored by FEDER funds through the program COMPETE - Programa Operacional Factores de Competitividade - and by national funds through FCT - Fundação para a Ciência e a Tecnologia -, under the project PEst-C/EME/UI0285/2013.

[1] Rao, C. N. R. et al., Chemphyschem, 2, 78-105, 2001.

[2] Ajayan, P. M and Tour, J. M. et al, Nature, 447, 1066-1068, 2007. 


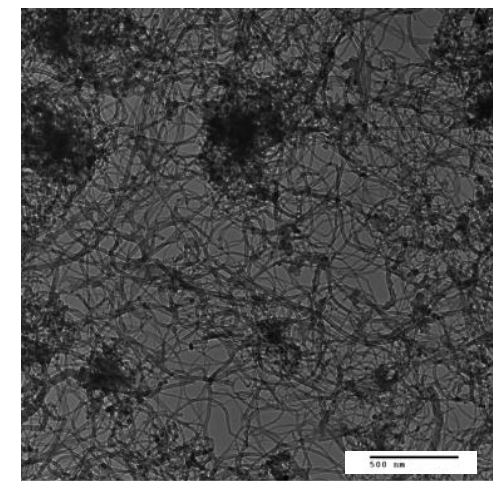

(a)

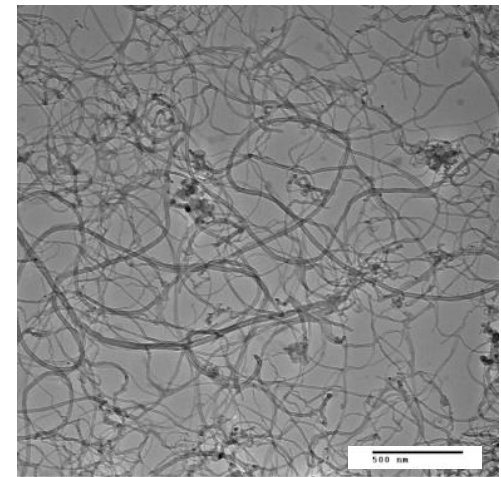

(b)

Figure 1. TEM images of CNT: (a) as-received and (b) after dispersion treatment.

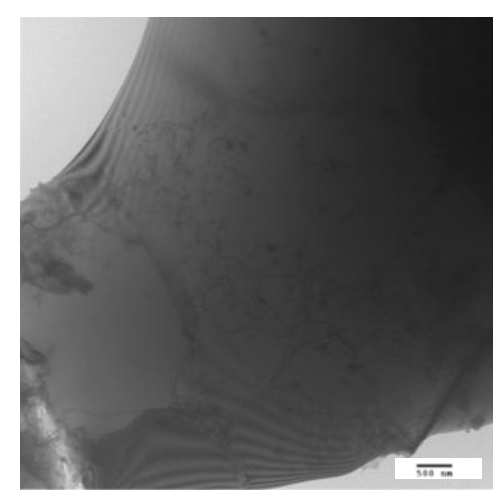

(a)

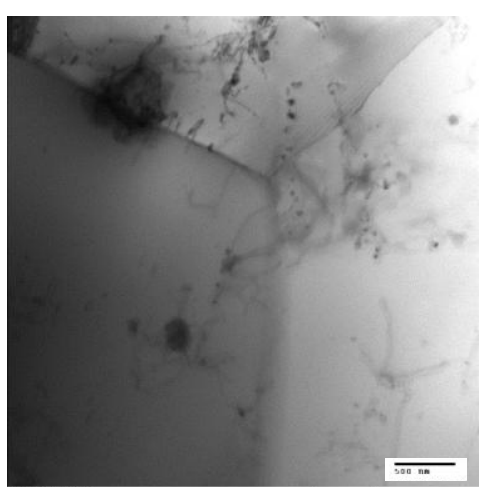

(b)

Figure 2. TEM images of CNT/Al nanocomposites with: (a) 0.75 wt. \% and (b) $1.0 \mathrm{wt} . \%$ of CNT.



(a)

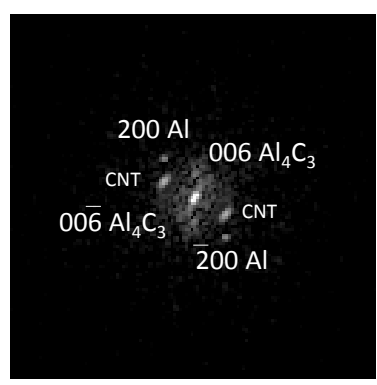

(b)

Figure 3. HRTEM image of nanocomposites shown a CNT embedded in the aluminum matrix. 